\title{
Implementando la instrucción diferenciada en el aula de clase de idioma extranjero
}

\section{implementing differentiated instruction in the foreign language classroom}

\author{
MSc. Heidi Marriott Toledo \\ Universidad Internacional del Ecuador, Ecuador \\ Universidad de Guayaquil, Ecuador \\ MSc. Ana María Zambrano García \\ Universidad de Guayaquil, Ecuador
}

Autor para correspondencia: hemarriottto@uide.edu.ec; heidi.marriottt@ug.edu.ec; ana.zambranog@ug.edu.ec

Fecha de recepción: 17 de Noviembre de 2017 - Fecha de aceptación: 15 de Mayo de 2018

Resumen: En la actualidad la enseñanza del inglés se hace cada vez más necesaria, sin embargo, el docente se enfrenta a grupos heterogéneos en sus clases, con diferentes niveles de conocimiento en inglés, lo que ocasiona la necesidad de aplicar metodologías proactivas que atiendan a toda la diversidad estudiantil al aprender. Es así como la Instrucción Diferenciada (ID) en el idioma extranjero influye en el proceso de enseñanza aprendizaje del inglés al permitir que el docente integre decisiones instruccionales informadas en las distintas formas de aprender, estilos de aprendizaje y las diferencias individuales para poder aplicar actividades adecuadas para cada tipo de alumno. El objetivo de esta investigación es proporcionar bases teóricas que confirmen que la aplicación de la instrucción diferenciada pueda resolver los problemas de heterogeneidad en las clases de inglés. Para esto, primero se proporcionarán antecedentes teóricos para identificar similitudes que establezcan buenas prácticas de este modelo. Luego, un análisis descriptivo proveerá información acerca de la composición diversa de los alumnos que pueden beneficiarse con la instrucción diferenciada y finalmente se presentara una forma práctica de aplicar la instrucción diferenciada según los estándares para la enseñanza de inglés en el Ecuador.

Palabras Claves: instrucción diferenciada; inglés como lengua extranjera; aprendizaje de inglés Abstract: Currently, English learning is more necessary than ever before. However, teachers often face mixed skills clases, with different English levels, which generates the need to apply proactive methodologies geared to tackle all this diversity while learning. This is how Differentiated Instruction (DI) in foreign language influences the teaching process allowing the teacher to integrate in the teaching learning process informed instructional decisions in the different ways of learning, learning styles, and individual differences in order to apply adequate activities for every student. The objective of this research is to provide the theorethical framework that confirm that the application of DI in the language classroom can solve the problems of heterogeneity in English classes. To achieve this, first, the theorethical background will be provided to identify similarities that determine good practices for this model. After that, a descriptive analysis will provide information about the diverse configuration of students who can benefit from DI, and finally, the differentiated application of this model in a specific skill will be presented according to the standards for English instruction in Ecuador.

Key Words: differentiated instruction; english as a foreign language; english learning 


\section{Introducción}

Los altos niveles de diversidad del cuerpo estudiantil en las aulas de inglés como Idioma Extranjero EFL y el variado rango de características que los mismos traen a la escuela han creado la necesidad de docentes que puedan establecer ambientes de aprendizaje que respondan asertivamente a las necesidades individuales, antecedentes, estilos de aprendizaje, habilidades y niveles de desempeño de los estudiantes más necesarios que nunca.

Las expectativas planteadas a los estudiantes demandan de los docentes un nivel de respuesta que los pueda ayudar a enfrentar retos educativos que van más allá de la simple repetición de hechos, esto hace de la Instrucción Diferenciada (ID) una alternativa a la educación tradicional. De acuerdo a Heacox (2012), este tipo de instrucción permite a los estudiantes demostrar lo que ellos pueden hacer en formas que toman ventaja de sus propias fortalezas, habilidades e intereses personales. Es una forma de efectivamente impartir instrucción a los estudiantes proveyendo una variedad de oportunidades de aprendizaje y por lo tanto enriqueciendo el frecuente currículo distrital sobre regulado, estándares y sobre todo los ambientes de evaluación represivos.

La instrucción diferenciada en los ambientes de clase es una oportunidad para mejorar el desempeño escolar a través de la implementación de oportunidades de aprendizaje innovadoras y que ofrecen retos a través del contenido, proceso y el producto mientras se usa la información obtenida a través de las distintas evaluaciones del conocimiento de los estudiantes y niveles de preparación, así como su información académica y antecedentes. Particularmente en el caso de la educación de inglés como idioma extranjero, la variedad de características estudiantiles poseen presentan demandantes retos a los docentes que, sumados a clases sobrepobladas, falta de recursos y la limitada exposición al lenguaje meta, afectan la calidad del aprendizaje de inglés.

El principal propósito de esta investigación será proveer un marco teórico que afirme el uso de la instrucción diferenciada como una alternativa viable para resolver estos problemas. Para responder a esto, los siguientes temas serán discutidos: primero, un bosquejo de estudios sobre el modelo de instrucción diferenciada será elaborado para proveer antecedentes teóricos, así como para identificar puntos comunes entre sus hallazgos que determinen buenas prácticas para este modelo. Luego, un análisis descriptivo de la actual configuración del cuerpo estudiantil será provista para dar información sobre los estudiantes que se pueden beneficiar de la instrucción diferenciada. Finalmente, una aplicación práctica sobre como la educación diferenciada puede ser aplicada en alineación con los actuales estándares para la instrucción en inglés en Ecuador será provisto para mostrar la forma en que se puede convertir en una alternativa para atender las necesidades de los estudiantes diversos.

\section{Instrucción diferenciada (ID)}

\section{Propósito de la instrucción diferenciada}

Walqui y van Lier (2010) indicaron que existe una brecha entre como los estudiantes adquieren el lenguaje y el propósito para el cuál lo usan. Para los estudiantes de inglés a menudo luchan con el uso de inglés para tareas más avanzadas que las que son presentadas en actividades 
de conversación. En ocasiones, esta dificultad entre el lenguaje conversacional y el académico causa que los estudiantes se retrasen y pierdan la motivación necesaria para continuar involucrándose en el aprendizaje del idioma.

El rango de habilidades, destrezas e intereses de los estudiantes en constante aumento moldean la forma en que ellos se acercan al inglés, por lo tanto, la forma en la que los docents planean instrucción significativa es la clave para mejorar el proceso de adquisición del segundo idioma. En este aspecto, la instrucción diferenciada ofrece un nuevo modelo que toma en consideración los antecedentes que los estudiantes traen al aula de clase, sus niveles de destrezas, estilos de aprendizaje e intereses para poder integrarlos en el aprendizaje del idioma y hacer la entrega de contenido, proceso y producto final del aprendizaje de los estudiantes más interesante y significativo.

\section{Rol del docente en la instrucción diferenciada}

Heacox (2012) indicaron que los docentes juegan un rol importante en la implementación del proceso de diferenciación. Debe ser su principal preocupación el convertirse en un facilitador que motive el aprendizaje de los alumnos en formas que ofrecen tanto retos como al igual que ofrecer una variedad de oportunidades para distintas formas de aprendizaje y las formas en las que alumnos presentan el resultado final de su aprendizaje. Los docentes deben tener en consideración las preferencias estudiantiles y sus necesidades educativas para entregar instrucción responsiva.

Como Alavinia and Farhardy (2012) indican cada clase incluye una variedad de características individuales, pero ciegamente creer en esas diferencias no es suficiente si los profesores no adaptan sus métodos instruccionales para cubrir las necesidades de sus alumnos. El estudio que ellos llevaron a cabo se enfocó en la forma en que los estudiantes con distintas características de aprendizaje aprendían vocabulario y como incluir estas diferencias para aproximarse a sus estilos individuales de aprendizaje mientras se ponía en práctica un programa de instrucción diferenciada para ayudarlos a adquirir exitosamente vocabulario. Los investigadores intuyeron los salones de clase se están volviendo cada vez más diversos, por lo tanto, los docentes deben sentirse dirigidos a estar preparados para atender distintos niveles de proficiencia y habilidades individuales para ayudar a los estudiantes obtener mejores resultados. Un área que los autores enfatizan en el estudio es la necesidad del alto nivel de respuesta de los docentes es adquirida a través de la práctica y permite ganar confianza en los pormenores del modelo.

\section{¿Cómo funciona la instrucción diferenciada?}

Como Rock, Gregg, Ellis y Gable (2008) indicaron, el modelo de instrucción diferenciada está basada en cuatro principios básicos. Primero, se enfoca en las destrezas importantes y en las ideas de áreas de contenido. Segundo, pone énfasis en la capacidad de respuesta a las necesidades individuales. Tercero, busca la integración de prácticas de enseñanza y evaluación centradas en el alumno y finalmente, planea para la modificación continua de contenido, proceso y productos en formas que satisfacen las necesidades individuales. Aunque la implementación de la diferenciación ha probado ser útil en el aula de clase, no está libre de críticas. Wormeli (2005) 
discutió esta oposición indicando la forma en que diferenciación otorga a ciertos alumnos una carga desigual, como estudiantes deben demostrar sus conocimientos ha debido tiempo, o aguantar prácticas de enseñanza caducas para ser capaces de competir en el mundo actualizado y estar preparados para rendir pruebas de alto-riesgo que evitan la individualización. Este estudio mostró una justificación a cada una de esas aseveraciones indicando que en muchos casos implementar la ID fue la diferencia que ayudó a los estudiantes a involucrarse y hacerse más responsables de su propio conocimiento.

La diferenciación no es carente de retos. Como Rock et al. (2008) discutieron, los docentes necesitan prestar especial atención a la información obtenida a través de las evaluaciones en clase para poder responder al desempeño de los alumnos y ajustar su plan de instrucción a tiempo. Los docentes deben estar conscientes que la evaluación es un aspecto importante, pero no el que debe guiar principalmente la instrucción. La evaluación debe ser enfocada como un proceso continuo que permita al docente implementar cambios en los planes de instrucción cuando sea necesario e introducir formas múltiples de evaluación auténtica e informal que ayuden a los alumnos a poner en uso sus habilidades para beneficiar y mejorar el aprendizaje mientras demuestran el conocimiento adquirido. Más aún, los docentes necesitan ser capaces de manejar las demandas de los creadores de políticas y oficiales distritales para cubrir el contenido y proveer práctica para pruebas estandarizadas y marcar un paso que beneficie el ambiente de aprendizaje. Asumir que involucrar a los alumnos en instrucción diferenciada los llevará a estar mal preparados para enfrentar evaluaciones y problemas de la vida real es infundado.

\section{Beneficios de la instrucción diferenciadas para estudiantes de idioma inglés}

La diferenciación permite a los estudiantes usar su potencial en cada tarea que enfrentan, esto los ayuda a involucrarse en procesos cognitivos más altos que tan solo desarrollar tareas diseñadas por el docente. Según Moss, Lapp and O'Shea (2011), los estudiantes requieren apoyo para involucrarse en aprendizaje de idiomas, éste apoyo puede ser encontrado en el uso de materiales diferenciados que pueden ayudar al estudiante a crear lenguaje más académico para participar activamente en más desafiantes tareas de lenguaje. Los estudiantes de idioma inglés (ELLs) usualmente necesitan ganar la suficiente proficiencia en el idioma para dominar más demandante contenido del curso. El uso de ID permite al docente proveer recurso que pueden ayudar a los estudiantes a hacer la transición de lenguaje comunicativo simpe y alcanzar el discurso académico requerido para llevar a cabo tareas más complicadas. Debido a que los hogares de los estudiantes no proveen las oportunidades para la práctica de inglés, las escuelas deben ayudarlos a superar esta brecha y proveer cambios para que ellos se involucren en práctica del lenguaje significativa.

El uso, por ejemplo, de textos por niveles en una alternativa para la práctica literaria diferenciada. Los estudiantes pueden usar textos que están alineados con su propio nivel lector y de habilidad y luego complementarlo con el uso de material adicional que aumente su experiencia lectora. Estos tipos de texto pueden ayudar a los estudiantes a moverse de lenguaje común a más avanzadas niveles de lenguaje. Esto ayuda el progreso de los estudiantes en su continuidad de aprendizaje y a su vez les permite adquirir destrezas de lenguaje más 
cuidadosamente, permitiéndoles involucrarse en práctica más demandante y construir las bases que pueden beneficiar múltiples áreas del proceso de aprendizaje de inglés.

La instrucción diferencia busca la integración de todos los niveles de destrezas presente en salones diversos para beneficiar el proceso de aprendizaje. Incorporando los antecedentes estudiantiles, intereses, estilos y necesidades de aprendizaje. No es una práctica fácil, pero es largamente soportada por investigación sobre sus insuperables beneficios para el aprendizaje de idiomas. Su éxito radica en la preparación de docentes y en sus niveles de respuesta. La diferenciación es una tarea demandante, pero es una que llevada a cabo apropiadamente puede rendir beneficios y ayudar a estudiantes a perseverar en su progreso en inglés.

\section{Características de los estudiantes ecuatorianos}

Los estudiantes ecuatorianos forman parte de uno de los grupos más culturalmente dinámicos en el Sistema educativo. Varios factores contribuyen a crear esta mezcla que se puede encontrar en los salones de clase públicos de Ecuador, entre estas se puede incluir la migración, las oportunidades de trabajo, prestigio escolar y últimamente el acceso masivo a educación gratuita que a menudo carga promesas de mejores oportunidades educativas. Muchos de los estudiantes que van a la escuela en grandes ciudades, como Guayaquil o Quito, se han transferido de escuelas en su mayoría porque sus padres necesitaron reubicarse para aceptar ofertas de trabajo. La migración de una ciudad a otra y mudanzas relacionadas a oportunidades de trabajo son a menudo atraídos a cambiar a sus hijos en instituciones tradicionales que son conocidas por su prestigio y han adquirido una reputación de excelencia a través de los años. Últimamente, un nuevo fenómeno está ocurriendo, padres esperando que sus hijos asistan a más modernizadas y remodeladas escuelas tradicionales, que poseen tecnología actual e infraestructura nueva, han empezado a buscar formas de transferir a distritos escolares que les garanticen un puesto.

La distribución escolar está determinada por el lugar donde los estudiantes viven, éste proceso ha sido altamente criticado y atraído muchas quejas debido a que muchos estudiantes tienen que viajar largas distancias entre distritos para asistir a la escuela. Sin embargo, cuando los estudiantes reciben cupo éstas escuelas transporte es raramente un problema. En estos casos, los padres se hayan en su mayoría dispuestos a transportarlos, proveer transporte escolar o público ya que el mismo no se encuentra incluido en la gratuidad.

De acuerdo con información recogida por el Instituto Nacional de Estadísticas y Censos INEC en el censo 2010, el 74.20\% de la población estudiantil asiste a instituciones públicas y sus niveles de asistencia en años se ha incrementado de 6,61 a 9,04 años en el 2010. Las tasas de analfabetismo en grupos de edad escolar también registran su más bajo nivel con $1.30 \%$ en el rango de 5-19 años, lo que podría significar que los esfuerzos realizados para proveer acceso a la educación a grupos críticos han rendido buenos resultados. Las más altas tasas de analfabetismo se pueden encontrar en poblaciones indígenas con un 20,4\% y mestizos con $12,9 \%$. Estas cifras posan una mayor preocupación para los oficiales educativos que necesitan proveen atención especial a estos grupos de estudiantes, más susceptibles a abandonar la escuela. 
Las escuelas más emblemáticas o tradicionales esta ubicadas en áreas urbanas, pero en los últimos años ha habido muchos intentos en ampliar el acceso a la educación para los niños que viven en las zonas menos privilegiadas de las grandes ciudades y los que pueden ser considerados de barrios de riesgo. Más del 67,1\% de área urbana y el $84.8 \%$ de los mismo son de instituciones que ofrecen educación inicial, básica o secundaria.

Las escuelas tradicionales usualmente sirven a una población muy extensa, con clases que pueden ir desde los 45 hasta 80 estudiantes dependiendo del número de aulas disponibles. Estas escuelas usualmente trabajan en jornadas dobles, en la tarde los alumnos de de los últimos años de Educación General básica o primaria superior y en la mañana los alumnos de Bachillerato.

Las clases son usualmente llevadas a cabo en edificios espaciosos con poca ventilación e iluminación. En la mayoría de los casos, careciendo de recursos didácticos. El número de salones usualmente varía dependiendo del número de alumnos que han sido derivados a través de la asignación distrital. Esto es más evidente en los últimos años donde más estudiantes estudian en espacios más pequeños y menos espacio está disponible para circular en el aula. La planta docente esta usualmente formada por docentes que poseen un título de tercer nivel y en algunos casos incluso maestrías y doctorados, los cuales instruyen a los alumnos en las materias determinadas por las guías curriculares.

De acuerdo a las guías curriculares para la Enseñanza del Idioma Inglés, se espera que los estudiantes del sistema educativo ecuatoriano alcancen un nivel B1 a lo largo de los seis años de instrucción en alineación con el Marco Común de Referencia Europeo para lenguajes: Aprendizaje, enseñanza y evaluación. Este proceso se debe alcanzar en incrementos de dos años en los cuales los estudiantes deben transicionar entre nivel de uso básico del idioma (A1-A2) y uso independiente (B1). Se espera que los docents trabajen con miras hacía el desarrollo de todas las destrezas, así como ayudar a los estudiantes a alcanzar los niveles de uso de lenguaje requeridos para la finalización de ese período. (Ministerio de Educación de Ecuador, 2012)

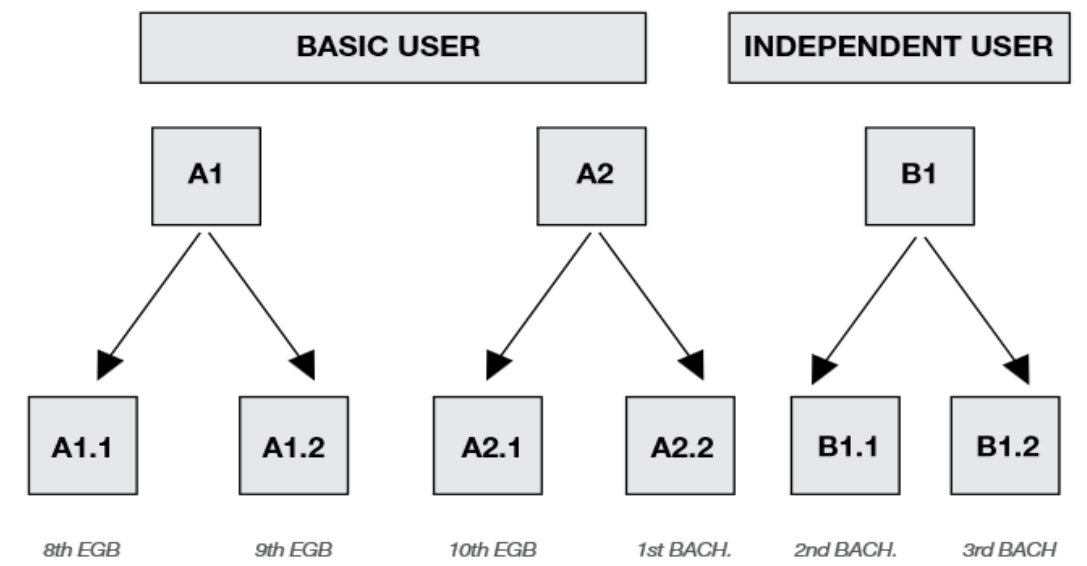

Figura 1. Niveles de proficiencia según Guías Curriculares para la enseñanza del Inglés Fuente: Ministerio de Educación (2012)

Los antecedentes estudiantiles en escuelas ecuatorianas son diversos, en parte debido a reglas para beneficiar a estudiantes de alto desempeño y comunidades en riesgo o desatendidas. La presencia de distintos grupos étnicos también ha sido identificada en el aula. Estudiantes de 
ascendencia blanco, afro ecuatoriana, indígena y mestiza pueden ser encontrados en dichos salones Los estudiantes de ascendencia indígena, que comprenden más de 14 diferentes grupos étnicos y más de 40 nacionalidades y los estudiantes mestizos constituyen el grupo más grande. No hay cifras actuales acerca de la configuración demográfica en las escuelas disponible para los docentes debido a la falta de registros públicos en las escuelas y en las oficinas educativas.

Los estudiantes con dificultades de aprendizaje carecen de diagnóstico previos. En la mayoría de los casos, la derivación docente al departamento de consejería estudiantil DECE es usualmente lo que genera atención a los casos particulares de disabilidad y a la identificación de necesidades educativas. A su vez la escasez de staff en este departamento, la mayoría de los estudiantes no interactúa con sicólogos escolares a menos que estén experimentado problemas de comportamiento. Adicionalmente, a pesar de que el Sistema ecuatoriano garantiza el acceso a educación que satisfaga las necesidades de los estudiantes, en muchos casos no hay diseñados protocolos previos para manejar casos de estudiantes con capacidades especiales o físicas. Debido a esto, son a menudo promovidos a los cursos inmediatos superiores sin haber alcanzado mayor avance o habiendo creado suficiente información para los futuros docentes puedan trabajar. Varios de estos grupos de estudiantes generan mayores demandas en docentes y autoridades escolares ya que han sido identificados como con mayor riesgo de abandonar la escuela como lo determina la información censal.

A pesar de que a menudo, la instrucción previa no prepara a los docentes para efectivamente atender todas estas necesidades, el contenido revisado sobre Instrucción Diferenciada provee una nueva perspectiva para entender y planear la instrucción de mejor forma y teniendo los perfiles estudiantiles en mente.

\section{Instrucción diferenciada en Ecuador}

\section{Estándares para la enseñanza de inglés como lengua extranjera EFL}

La instrucción de inglés ecuatoriana está alineada con el Marco Común Europeo de Referencia para Lenguajes: Aprendizaje, enseñanza y evaluación (MCER). Los estándares para el idioma inglés dictan que para el año 2017, los docentes deben trabajar para lograr que los estudiantes adquieran el nivel B1 para el final de su educación media (Ministerio de Educación del Ecuador, 2012a). Ecuador ha implementado un sistema continuo para reforzar la instrucción de inglés desde el 2011. Este proceso incluye la provisión de textos oficiales, entrenamiento y evaluación de los docentes en servicio, así como la contratación de nervios y actualizados docentes en colaboración con un programa de becas del Ministerio de Educación y el organismo que rige la educación superior Senescyt.

El Segundo punto en este proceso ha ocupado la mayor parte del tiempo de los docentes en servicio durante los últimos tres años. En las etapas finales del proceso de evaluación, los docentes deben acreditar al menos un nivel B2 de acuerdo con el MCER. Para ello requieren tomar pruebas oficiales como el Toefl iBT para probar su proficiencia en el idioma. Este requisito ha causado en los docentes altos niveles de estrés, ya que muchos de ellos fueron ya evaluados y reprobaron. Luego de asistir a una capacitación de un año, serán nuevamente evaluados que será según se indica su última oportunidad antes de arriesgar perder sus puestos de 
trabajo. Los nuevos docentes que entran al Sistema también tienen este requisito, pero lo pueden probar con más pruebas que las anteriormente mencionadas. Los estándares de calidad son nuevos para la educación ecuatoriana y aunque las autoridades han vuelto su misión hacer que se sigan, los mismos han tomado un segundo plano debido a los intereses personales más apremiantes de los docentes. Este hecho sienta un precedente en la importancia que los estándares han alcanzado en la educación ecuatoriana, ya que, aunque su uso es obligatorio el mismo no es realmente supervisado.

\section{Formas de diferenciar la instrucción}

Para poder modelar cómo funcionaría la instrucción diferenciada ID en el aula de inglés como idioma extranjero un estándar del perfil de salida en la destreza de lectura ha sido escogido, el mismo que debe haberse alcanzado para la finalización de Octavo grado, y que está incluido en el nivel A1.1. De acuerdo con las guías curriculares para este nivel, los estudiantes deben ser capaces de "entender e identificar textos informativos simples (e.g. etiquetas, mensajes, postales, catálogos, etc.) frase por frase durante un tiempo y a su vez ser capaces de comprender nombres familiares, palabras y frases básicas y volver a leer como se requiere, extraer la idea principal e información clave de textos informativos simples." (Ministerio de Educación del Ecuador, 2012b, p. 13).

Este estándar implica que los estudiantes deberían ser capaces de entender textos simples en el idioma inglés. Estos son textos cuyo formato provee información en una forma que permite la identificación de puntos clave y hace más fácil a los estudiantes encontrar la información. Algunos ejemplos de este tipo de textos pueden ser libros reales, historia, autobiografías entre otros. Los estudiantes deben ser capaces de reconocer la información requerida y obtener su significado.

Tomlinson (1999) indicó que, para diferenciar instrucción efectivamente los docentes tienen que fomentar el aprendizaje a través del uso de planes fuertes y confiables que consideren las diferencias individuales de los estudiantes. Para alcanzar este objetivo, los instructores EFL deben considerar los siguientes aspectos en su plan de instrucción:

\section{Contenido}

El currículo es una de las partes más importantes de la instrucción ya que guía el plan instruccional del docente. Los temas escogidos para ser parte del currículo deben por lo tanto deben permitir al estudiante crear una adecuada base de conocimiento. Como Heacox (2012) sugiere, el currículo debe incluir conceptos relevantes y esenciales, destrezas, procesos y también debe considerar el gradual aumento del nivel de complejidad.

Para diferenciar el contenido, el primer paso será pre-evaluar el conocimiento y nivel de destreza de los estudiantes. Esto proveerá información sobre los temas que ya son dominados y que sólo se deben repasar y los que requieren énfasis más fuerte durante clases, Para los educadores EFL, tener esta información les permitirá entender el nivel de destreza que los estudiantes poseen para entender ese tipo de texto. Como una forma de entender que conocimiento poseen los estudiantes sobre esos textos, se puede proveer a los alumnos de 
diferentes textos en mapas conceptuales. Herrera, Morales, and Murry (2013) indican que los mapas conceptuales permiten a los estudiantes demostrar su conocimiento haciendo uso de representaciones lingüísticas y no linguiísticas. Esta es una importante forma de aproximar el tema ya que de esa forma no se desalienta al estudiante mientras aprende inglés.

\section{Proceso}

El proceso de la instrucción diferenciada debería reflejar la información recogida en los perfiles de aprendizaje e intereses de nuestros estudiantes. Este proceso puede comprender distintos niveles de complejidad para ayudar a los estudiantes a alcanzar niveles más altos de pensamiento formal operativo. Como Heacox (2012) sostiene, los docentes que diferencian su instrucción deberían considerar la taxonomía de Bloom y la teoría de inteligencias múltiples de Gardner pues ellas proveen las bases sobre como los alumnos aprenden mejor y permiten niveles adecuados de desafío académico y entregando instrucción direccionada con actividades de más alto nivel cognitivo.

Un ejemplo durante esta etapa en el caso de la destreza lectora es el uso de los textos por niveles. De acuerdo con lo que indica Moss et al. (2011) los textos de lectura por niveles permiten a los estudiantes de inglés el relacionarse con textos que toman en consideración sus necesidades lectoras mientras les proveen niveles adecuados de desafío y los apoyan en continuar profundizando su conocimiento.

\section{Producto}

Los estudiantes deben ser capaces de demostrar los resultados finales de su aprendizaje mostrando lo que han aprendido de una forma tangible. Como Heacox (2012) expresó, productos deben ser capaces de reflejar las cosas que los estudiantes han entendido y son capaces de aplicar. Tomando en consideración niveles de desafío y los perfiles de aprendizaje debe ser una parte importante de los productos ya que los estudiantes deben ser capaces de demostrar sus fortalezas al igual que tener la oportunidad de involucrarse en actividades desafiantes.

Como Pham (2012) sostuvo, los productos deberían usar distintas formas de evaluación y son la mejor forma de proveer retroalimentación que enriquezca el aprendizaje de los estudiantes. Los docentes que efectivamente involucren y proveen a los estudiantes comentarios positivos y constructivos sobre su trabajo tendrán más posibilidades de interesarlos en trabajo cooperativo e independiente en el futuro. Este es muy importante para estudiantes de inglés como lengua extranjera ya que carecemos del ambiente necesario para el desarrollo del lenguaje. Si se entrega retroalimentación, los estudiantes pueden sentirse más motivados para incrementar su práctica del lenguaje fuera de los límites de la escuela.

Proveer a los estudiantes con un menú de proyectos puede ser una gran alternativa para que ellos se involucren activamente en sus trabajos finales. No solo tendrán varias formas de demostrar sus resultados de aprendizaje, sino también estos productos considerarán sus múltiples inteligencias y variados niveles de taxonomía para ofrecer desafío. La posibilidad de monitorear el tipo de actividad escogida permitirá al docente ayudarlos a alcanzar mayores retos académicos. Realizar entrevistas también permite a los estudiantes demostrar su comprensión de 
un particular texto. Herrera et al. (2013) indica que cuando los estudiantes se involucran en la evaluación de su propio trabajo, ganas más entendimiento sobre cómo crear productos de alta calidad. Trabajar con sus pares les permite a los alumnos sentirse más cómodos. Finalmente, el uso de rúbricas como indica Lin también puede ser implementado para que ellos se informen sobre los criterios que involucran productos de alta calidad.

\section{Ambiente de aprendizaje}

Para crear un apropiado ambiente de aprendizaje Gibson (2010) afirma que, el éxito de la instrucción diferenciada puedo solo ocurrir si procedimientos y rutinas son establecidas para ayudar a los alumnos a desarrollar comportamientos que apoyen su efectiva participación en clase. El autor desarrolló cinco pasos para este efecto. Dichos pasos incluyen establecer el ambiente físico de la clase, usar la información obtenida para informar la práctica, manejar los recursos disponibles, crear los procedimientos de clase y rutinas y finalmente, proveer educación y práctica de calidad.

\section{Conclusión}

La instrucción tradicional ha probado carecer de la posibilidad de atender las necesidades en aumento de los cuerpos estudiantiles en todos lados. La instrucción de inglés como idioma extranjero es una delicada tarea que requiere que los docentes encuentren y creen oportunidades reales para hacer realizar conexiones significativas al contenido escolar. Esta meta no puede ser alcanzada si como educadores no tomamos el tiempo para impartir instrucción de una forma que le resulte relevante a nuestros estudiantes. Para alcanzar esto, el modelo de instrucción diferenciada ofrece la posibilidad de usar las fortalezas, habilidades y niveles de preparación y otras preferencias de aprendizaje para beneficiar su aprendizaje del idioma inglés.

Recrear un ambiente efectivo de inglés como idioma extranjero puede ser una empresa intimidante si el docente no se toma el tiempo de evaluar estudiantes e identificar sus intereses. Conocer sus motivaciones es la clave para asegurar su involucramiento en clase y ayudarlos a mejorar sus habilidades de lenguaje. Diferenciar no significar hacer tareas múltiples sin propósito sino la oportunidad de ofrecer una variedad de un plan de instrucción específicamente dirigido que cubra las necesidades de los estudiantes que estamos enseñando.

Para los educadores ecuatorianos, la instrucción diferenciada brinda la oportunidad de incorporar en clase la diversidad que los estudiantes traen al salón de clase en una forma que les permita la construcción del conocimiento en una forma democrática y cooperativa. Sin embargo, el principal reto debe ser crear rutinas instruccionales claras que puedan proveer estructura a nuestros estudiantes diversos y a su vez ayuden a guiar y modelar su aprendizaje.

Los docentes tienen la gran responsabilidad de hacer la diferenciación posible. Para esto primero, deben reconocer las diferencias de los estudiantes y como se constituyen sus necesidades educativas. Usando esta información se debe desarrollar un plan de instrucción que genere involucramiento y presente retos en un contexto contextualizado. Finalmente, deben proveer un sistema de soporte que favorezca la interacción estudiantil para el efectivo uso del lenguaje dentro y fuera de la clase. La instrucción diferenciada ciertamente requiere práctica, 
pero más que eso, requiere compromiso. Es un proceso que necesita ser perfeccionado con el pasar del tiempo, pero si no se toma el primer paso estaremos evitando que los estudiantes tengan la posibilidad de apropiarse de su propio aprendizaje de inglés y usarlo efectivamente para su beneficio.

\section{Bibliografía}

Alavinia, P., \& Farhady, S. (2012). Teaching vocabulary through differentiated instruction: Insights from multiple intelligences and learning styles. Modern Journal of Language Teaching Methods, 2(4), 73-90. Retrieved from http://search.proquest.com.er.lib.kstate.edu/docview/1429385932?accountid=11789

Gibson, V. (2010). Differentiating instruction: Teaching differently to improve student outcomes. Retrieved from http://www.compasslearning.com/files/docs/1252WP-DIFINST-1110WEB.pdf

Heacox, D. (2012). Differentiating instruction in the regular classroom: How to reach and teach all learners. Minneapolis, MN: Free Spirit Publishing.

Herrera, S., Morales, R., \& Murry, K. (2013). Assessment accommodations for classroom teachers of culturally and linguistically diverse students (2nd ed.). Boston: Pearson.

Instituto Nacional de Estadisticas y Censos [National Institute of Statistics and Census]. (2010). Ecuador en cifras [Ecuador in numbers]. Quito, Ecuador: Author. Retrieved from http://www.ecuadorencifras.gob.ec/wpcontent/descargas/Presentaciones/capitulo_educacion_censo_poblacion_vivienda.pdf

Ministerio de Educación del Ecuador [Ministry of Education of Ecuador]. (2012a). Estándares de calidad educativa [Standards of educational quality] Ecuadorian in-service English teaching standards - English language learning standards. Quito, Ecuador: Author. Retrieved from http://educacion.gob.ec/wpcontent/uploads/downloads/2012/09/estandares_2012_ingles_opt.pdf

Ministerio de Educación del Ecuador [Ministry of Education of Ecuador]. (2012b). National English curriculum guidelines. Quito, Ecuador: Author. Retrieved from http://educacion.gob.ec/wpcontent/uploads/downloads/2013/09/Curriculum_Guidelines_EFL_100913.pdf

Moss, B., Lapp, D., \& O'Shea, M. (2011). Tiered texts: Supporting knowledge and language learning for English learners and struggling readers. English Journal, 100(5), 54-60. Retrieved from http://search.proquest.com.er.lib.kstate.edu/docview/864566175?accountid=11789

Pham, H. L. (2012). Differentiated instruction and the need to integrate teaching and practice. Journal of College Teaching \& Learning, 9(1), 13-20. 
Peralta-Nash, C., \& Dutch, J. A. (2000). Literature circles: Creating an environment for choice. Primary Voices $K$ - 6, 8(4), 29-37. Retrieved from http://search.proquest.com.er.lib.kstate.edu/docview/221703895?accountid=11789

Rock, M. L., Gregg, M., Ellis, E., \& Gable, R. A. (2008). REACH: A framework for differentiating classroom instruction. Preventing School Failure, 52(2), 31-47. Retrieved from http://search.proquest.com.er.lib.k-state.edu/docview/228486162?accountid=11789

Tomlinson, C. (1999). The differentiated classroom: Responding to the needs of all learners. Alexandria, VA: Association for Supervision and Curriculum Development.

Walqui, A., \& van Lier, L. (2010). Scaffolding the academic success of adolescent English language learners: A pedagogy of promise. San Francisco, CA: WestEd.

Wormeli, R. (2005). Busting myths about differentiated instruction. Principal Leadership, 5(7), 28-33. Retrieved from http://search.proquest.com.er.lib.kstate.edu/docview/234990154?accountid=11789 